\title{
Risk of Non-Alcoholic Fatty Liver Disease in Xanthelasma Palpebrarum
}

\author{
Hsuan-Wei Chen' \\ Jung-Chun Lin (D) \\ Ying-Hsuen $\mathrm{Wu}^{2,3, *}$ \\ Yi-Lin Chiu (D) ${ }^{4, *}$ \\ 'Division of Gastroenterology, \\ Department of Internal Medicine, Tri- \\ Service General Hospital, National \\ Defense Medical Center, Taipei, Taiwan; \\ ${ }^{2}$ Department of Ophthalmology, China \\ Medical University Hospital, China \\ Medical University, Taichung City, Taiwan; \\ ${ }^{3}$ School of Medicine, College of Medicine, \\ China Medical University, Taichung City, \\ Taiwan; ${ }^{4}$ Department of Biochemistry, \\ National Defense Medical Center, Taipei, \\ Taiwan
}

*These authors contributed equally to this work
Correspondence: Yi-Lin Chiu Department of Biochemistry, National Defense Medical Center, Taipei, Taiwan Email yc566@georgetown.edu

Ying-Hsuen Wu

Department of Ophthalmology, China

Medical University Hospital, China

Medical University

Email amywu04I4@gmail.com
Background: Xanthelasma palpebrarum (XP) is a sign of hyperlipidemia and is closely linked to atherosclerosis. Since fatty liver shares similar risk factors with atherosclerosis, we hypothesized that patients with XP are also at risk of non-alcoholic fatty liver disease (NAFLD).

Methods: In this retrospective cohort study, 37 patients with XP were compared with sexand age-matched controls undergoing general health examination. Moreover, demographic information and lipid profiles were compared. The risk of NAFLD was evaluated using the hepatic steatosis and ZJU indices. In addition, we analyzed publicly available RNA sequencing data from the GSE48452 and GSE61260 datasets in the Gene Expression Omnibus database.

Findings: Patients with XP had higher scores of hepatic steatosis index ( $37 \pm 1.13$ vs $32 \pm$ $0.82, p=0.0006)$ and ZJU index $(38.77 \pm 1.0$ vs $33.88 \pm 0.74, p=0.0002)$. In addition, they had higher levels of lipid parameters, including total cholesterol, low-density lipoprotein (LDL), and fasting glucose. Among patients with fatty liver, individuals presenting with XP showed higher serum levels of total cholesterol ( $216 \pm 10.4$ vs $188.9 \pm 7.6, p=0.04)$, fasting glucose $(117.1 \pm 6.4$ vs $98.3 \pm 2.4, \mathrm{p}=0.002)$, and low-density lipoprotein $(145.1 \pm 8.7 \mathrm{vs}$ $115.6 \pm 6.4, \mathrm{p}=0.009)$ than those without XP. In gene expression analysis, individuals presenting with non-alcoholic steatohepatitis showed higher $Z$ scores of xanthelasma than those without non-alcoholic steatohepatitis.

Conclusion: Our results suggest that individuals with XP have a higher risk of progression to NAFLD and develop a more severe dyslipidemia.

Keywords: xanthelasma, xanthoma, fatty liver, NASH, NAFLD

\section{Introduction}

Xanthelasma palpebrarum (XP) is a yellowish plaque usually seen on the eyelid with demarcation, which is associated with dyslipidemia. Approximately 9.1 to $67.9 \%$ of patients with XP have dyslipidemia. ${ }^{1}$ Therefore, XP not only affects the cosmetic appearance but also serves as a red flag for hyperlipidemia. Hyperlipidemia, defined as elevated low-density lipoprotein cholesterol (LDL) and reduced high-density lipoprotein cholesterol (HDL), is associated with atherosclerosis and cardiovascular diseases, $^{2}$ and patients with XP are at risk for premature atherosclerosis. ${ }^{3,4}$ Furthermore, systematic reviews and meta-analyses have confirmed a higher incidence of atherosclerosis in patients with XP. ${ }^{5}$ Despite having a normal lipid profile, patients with XP have a higher incidence of atherosclerosis. ${ }^{6,7}$ Since fatty liver disease is also a lipid-related disease, we hypothesized that the presence of XP might serve as a predictor of hepatic lipid accumulation. Therefore, in this study, 
we attempted to assess the risk of developing non-alcoholic fatty liver disease (NAFLD) in patients with XP.

$\mathrm{XP}$ is more prevalent in women than in men, with peak incidence occurring in the 40 - to 50 -year-old population group. ${ }^{8}$ In contrast, NAFLD is more prevalent in men than in women, and both the incidence and severity of the disease increase with age. ${ }^{9}$ Patients with NAFLD have a higher prevalence of obesity and metabolic syndrome; similarly, individuals with obesity, diabetes, dyslipidemia, and metabolic syndrome are at risk for NAFLD. ${ }^{10,11,12}$ In this study, 37 patients with XP were enrolled to evaluate the relationship between various physiological parameters and the percentage of XP patients with NAFLD. In contrast, liver microarray data of GSE48452 and GSE61260 datasets were used to investigate whether patients with NAFLD or non-alcoholic steatohepatitis (NASH) have a higher GSVA score of xanthelasma.

\section{Patients and Methods}

\section{Ethical Statement}

This study was approved by the Institutional Review Board (IRB), Tri-Service General Hospital (number C202005016). In this study, patient's personal information will not be seen and privacy was totally protected. Assessed as low risk, The IRB approved the abovenamed application for signature exemption on ICF and confirmed this research complied with the declaration of Helsinki. The IRB is organized and operated in compliance with the International Conference on Harmonization (ICH)/World Health Organization (WHO) Good Clinical Practice (GCP) and applicable laws and regulations.

\section{Subjects}

From January 2000 to December 2018, 37 patients diagnosed with XP were enrolled in this study. These patients were diagnosed by cardiologists, ophthalmologists, family physicians, plastic surgeons, and dermatologists. Participants in the control group were randomly selected from the health examination department. Exclusion criteria included a history of alcohol consumption, smoking, malignancy, and hepatitis virus infection. Age- and sexmatched controls were compared to the patients with XP.

\section{Study Design}

This study was a retrospective study. Body weight and height and comorbidities, such as coronary artery disease (CAD), type 2 diabetes mellitus, stroke, hypertensive cardiovascular disease, and fatty liver, were recorded for analysis. CAD refers to the reduction of blood flow to the cardiac muscle due to atherosclerosis in the arteries of the heart (Geneva: World Health Organization in collaboration with the World Heart Federation and the World Stroke Organization. pp. 3-18.). Patients with CAD may present chest pain or discomfort. In our study, the diagnosis of CAD was made based on the patient's symptoms, stress echocardiography results, cardiac biochemical markers (creatinine kinase and troponin-I), myocardial scintigraphy, or coronary angiography. All patients were evaluated by cardiologists. Laboratory data, including aspartate aminotransferase (AST), alanine aminotransferase (ALT), total cholesterol, LDL, fasting glucose, HDL, triglyceride, and hemoglobin A1c (HbAlc), were separately recorded. The hepatic steatosis index (HSI) ${ }^{13}$ and ZJU index ${ }^{14}$ were used to evaluate the risk of NAFLD. Liver fibrosis scores (FIB-4) were used to evaluate liver stiffness. FIB-4 score was calculated using the following formula: age (years) $\times$ AST $[\mathrm{U} / \mathrm{L}] /$ (platelets $[109 / \mathrm{L}] \times$ ALT $[\mathrm{U} / \mathrm{L}]) 1 / 2$, computed from laboratory results in all patients. Liver fibrosis was classified as low ( $<1.45$, no or minimal fibrosis), intermediate (1.45-3.25), and high (>3.25, significant fibrosis). Hypercholesterolemia was defined as total cholesterol $>$ $200 \mathrm{mg} / \mathrm{dL}$.

\section{Gene Expression Profiling}

The microarray data of GSE48452 published by Ahrens et $\mathrm{al}^{15}$ and GSE61260 published by Horvath et $\mathrm{al}^{16}$ were downloaded from the NCBI Gene Expression Omnibus (GEO) database (http://www.ncbi.nlm.nih.gov/geo/). These databases analyzed the difference in xanthelasma $Z$ scores between patients with or without NAFLD and NASH using whole gene expression profiling. The GSE48452 dataset contained data collected from 73 samples of human liver grouped into $\mathrm{C}$ (control=14), $\mathrm{H}$ (healthy obese $=27$ ), $\mathrm{S} \quad$ (steatosis $=14$ ), and $\mathrm{N}(\mathrm{NASH}=18)$ for analysis. The GSE61260 dataset contained data collected from 134 human liver samples from patients with morbid obesity and healthy controls for analysis. The maximum expression values of multiple probe IDs that corresponded to one official gene symbol were computed, and these values were used to represent the expression level of the related gene symbol.

The GSVA approach was developed by Hanzelmann et al. ${ }^{17}$ For the calculation of the xanthelasma GSVA score, DisGeNET was used to obtain a list of genes 
associated with xanthoma (MeSH ID:D014973). ${ }^{17}$ The genes were further screened by "Genetic Variation" of the association type. Eight genes were filtered out, including $\mathrm{ABCG} 5,{ }^{18,19}$ LDLRAP1, ${ }^{20}$ PCSK9,${ }^{21}$ APOA $1,{ }^{21}$ ALOX5 $,{ }^{22} \mathrm{ABCG} 8,{ }^{23,24} \mathrm{LDLR}^{25-27}$ and $\mathrm{APOE},{ }^{28}$ which have been reported to be associated with the development of xanthoma. The above genes were used to create the xanthelasma signature, and the GSVA scores were calculated using the GSVA package in $\mathrm{R}$ with default settings as previously described. ${ }^{29}$ Functional enrichment analysis was performed using the ToppGene Suite. ${ }^{30}$

\section{Statistical Analysis}

Continuous variables are presented as the mean \pm standard error of the mean (S.E.M). The results for categorical variables are expressed as percentages. Statistical comparisons between the two groups were performed using Student's $t$-test (unpaired $t$-test) or Chi-square test, according to the type of data. All statistical analyses were performed using GraphPad Prism 7.0 software (version 18.0; SPSS Inc., Chicago, IL, USA).

\section{Results}

\section{Flow Chart of the Study Design}

We initially evaluated the basic eligibility of 60 patients diagnosed with XP. Of theme, 23 patients were excluded because they were only underwent the cosmetic surgery and did not undergo any laboratory test. Forty-one patients with other diseases, but not XP, served as controls, and 37 patients with XP were eventually included in this study (Figure 1).

\section{Demographics and Baseline Features}

The baseline demographic characteristics of the patients are summarized in Table 1. A total of 37 patients with XP, 32 females (86.5\%), and 5 males (13.5\%), were consecutively enrolled. The mean age was $58.14 \pm 2$.0-years (range: 37-79 years). People from the health examination department who were age-and sex-matched were randomly included in the control group. People with XP displayed higher body weight $(65.62 \pm 1.7$ vs $60.13 \pm 1.75, \mathrm{p}=0.03)$ and higher body mass index (BMI) $(25.61 \pm 0.62$ vs 22.81 $\pm 0.51, \mathrm{p}=0.0009$ ). Serum lipid profiles were significantly different between the two groups. Patients with xanthelasma showed higher serum level of cholesterol (218.3 \pm 7.06 vs $189.8 \pm 6.54, \mathrm{p}=0.004)$, LDL $(142.9 \pm 6.15$ vs $116.2 \pm 5.32, \mathrm{p}=0.0015)$, and fasting glucose $(107.2 \pm 3.74$

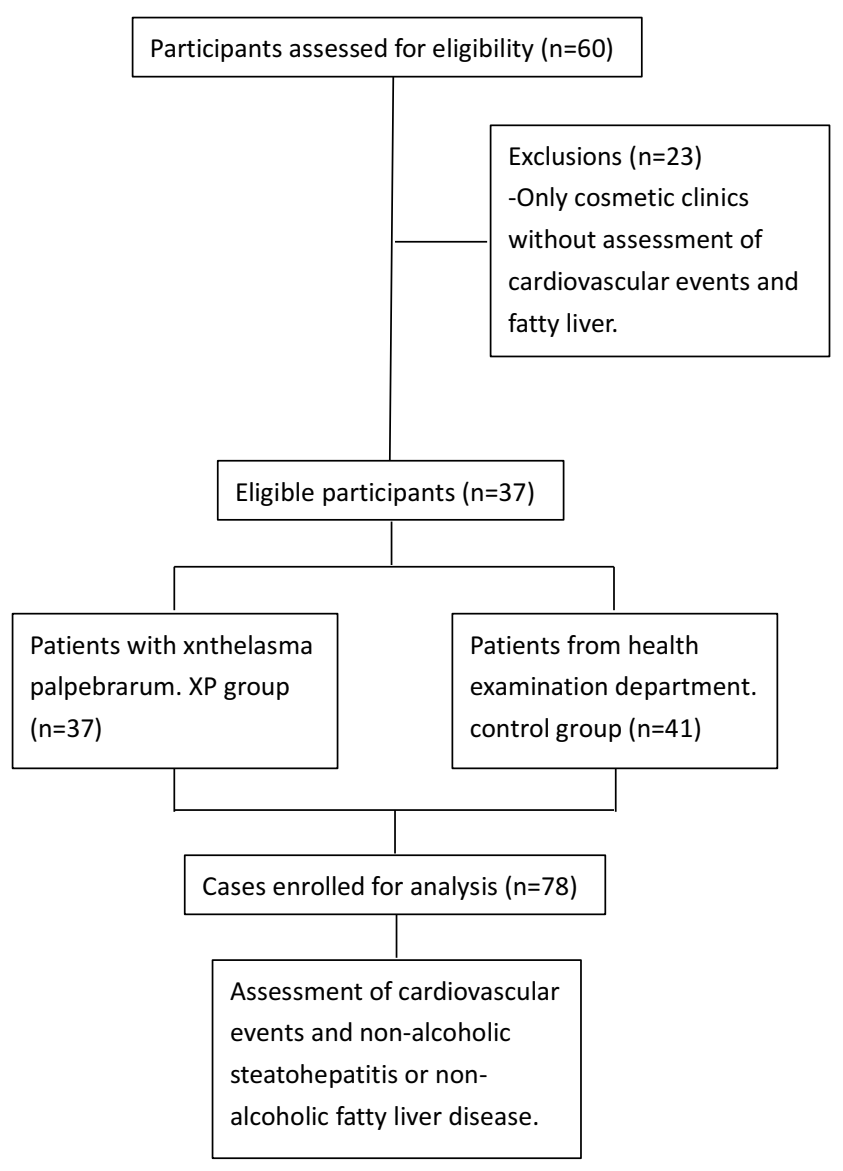

Figure I Flow chart of patient inclusion and exclusion. A total of 60 patients diagnosed with xanthelasma palpebrarum (XP) were initially assessed for enrollment in this study. Among them, 23 patients visited cosmetic clinics only and were excluded. Thirty-seven patients with XP were enrolled. Forty-one age- and sexmatched individuals from the health examination department were included in the control group.

vs $98.03 \pm 2.35, \mathrm{p}=0.038$ ). People in the XP group also presented with a higher prevalence rate of CAD, but no difference was noted for the prevalence of type 2 diabetes mellitus and stroke between the two groups. Regarding hepatitis markers and fibrosis scores (FIB-4), both groups showed similar conditions. However, the XP group showed higher HIS and ZJU scores, indicating the risk of NAFLD.

\section{Metabolic Diseases in Normal or Hyper-Cholesterolemia Subgroups}

Patients were classified into normal and hypercholesterolemia subgroups (Table 2). No difference was noted in age distribution, but patients with XP showed a significantly higher BMI than those in the control group. In addition, patients with XP showed a trend of a higher incidence rate of CAD but a similar incidence rate of type 2 diabetes mellitus and stroke. Even at normal serum cholesterol 
Table I Clinical Characteristics of Patients in Control and Xanthelasma Palpebrarum Groups

\begin{tabular}{|c|c|c|c|}
\hline & Control Group (n=4I) & XP Group $(n=37)$ & $P$ value \\
\hline Age (mean; IQR, years) & $54.22 ; 15$ & $58.14 ; 12$ & 0.1 \\
\hline Sex (female (n), \%) & $33,80.5 \%$ & $32,86.5 \%$ & 0.48 \\
\hline Body height (mean; IQR, cm) & $162.1 ; 10$ & $160.1 ; 12$ & 0.3 \\
\hline Weight (mean; IQR, kg) & $60.13 ; 16.8$ & $65.62 ; 13$ & $0.03 *$ \\
\hline Body mass index (mean; IQR) & $22.81 ; 3.59$ & $25.61 ; 2.83$ & $0.0009 *$ \\
\hline Coronary artery disease $(n)$ & 0 & 10 & $0.001 *$ \\
\hline Type 2 diabetes mellitus ( $n$ ) & 3 & 8 & 0.07 \\
\hline Stroke (n) & 0 & 0 & \\
\hline Total cholesterol (mean; IQR, mg/dL) & $189.8 ; 59.5$ & $218.3 ; 52$ & $0.004^{*}$ \\
\hline LDL (mean; IQR, mg/dL) & II $6.2 ; 44.5$ & $142.9 ; 47.5$ & $0.002^{*}$ \\
\hline HDL (mean; IQR, mg/dL) & $53.27 ; 17.5$ & $48.3 ; 14.5$ & 0.07 \\
\hline Triglyceride (mean; IQR, mg/dL) & $131.5 ; 63$ & $155.3 ; 113$ & 0.22 \\
\hline Fasting glucose (mean; IQR, mg/dL) & $98.03 ; 15.5$ & $107.2 ; 27.5$ & $0.03 *$ \\
\hline GOT (mean; IQR, U/L) & $22.88 ; 9$ & $25.07 ; \mathrm{II}$ & 0.52 \\
\hline GPT (mean; IQR, U/L) & $24.34 ; 15$ & $24.69 ; 16$ & 0.94 \\
\hline Hepatic steatosis index (mean; IQR) & $32 ; 7.6$ & $37 ; 7.36$ & $0.0006 *$ \\
\hline ZJU index (mean; IQR) & $33.88 ; 5.62$ & $38.77 ; 7.31$ & $0.0002 *$ \\
\hline FIB-4 score (mean; IQR) & I.I5; 0.6 & I.I8; 0.72 & 0.85 \\
\hline Low (FIB-4 <I.45, mean; IQR) & $0.98 ; 0.45$ & $0.77 ; 0.27$ & 0.12 \\
\hline Intermediate to high (FIB-4> I.45, mean; IQR) & $\mathrm{I} .84 ; 0.44$ & I.99; 0.92 & 0.9 \\
\hline
\end{tabular}

Note: Baseline data, including demographic parameters and laboratory data, are presented. ${ }^{*} \mathrm{p}<0.05$.

Abbreviations: LDL, low-density lipoprotein; HDL, high-density lipoprotein; GOT, aspartate aminotransferase; GPT, alanine aminotransferase; ZJU, Zhejiang University index; FIB-4, fibrosis-4 score; IQR, interquartile range.

levels, patients with XP still presented with a higher level of LDL $(111.55 \pm 4.18$ vs $100.14 \pm 2.99, \mathrm{p}=0.04)$. Compared to individuals with normal cholesterolemia in the control group, the hypercholesterolemia subgroup in XP showed higher scores of HSI and ZJU indices. Even in normal cholesterolemia, patients with XP presented with higher scores of the ZJU index $(37.65 \pm 1.52$ vs $32.97 \pm 1.19, \mathrm{p}=0.045)$.

\section{Comparison Between Patients with or without XP with Fatty Liver}

We compared the lipid profiles between the XP and nonXP patients with fatty liver. The diagnosis of fatty liver was based on sonographic findings. Patients with XP showed higher serum level of total cholesterol (216 \pm 10.4 vs $188.9 \pm 7.6 \mathrm{mg} / \mathrm{dL}, \mathrm{p}=0.04)$, fasting glucose $(117.1 \pm 6.4$ vs $98.3 \pm 2.4, \mathrm{p}=0.002)$, and LDL $(145.1 \pm$ 8.7 vs $115.6 \pm 6.4$ ) (Figure 2).

\section{Positive Relationship Between Xanthelasma and $\mathrm{NASH}$}

To ascertain the influence of steatohepatitis on the expression of xanthelasma-associated genes, we analyzed the metadata of GEO. The extent of the expression of xanthelasma-associated genes was presented with a $Z$ score. In GSE48452, people with steatohepatitis showed higher $Z$ scores than those in the control group $(p=0.015)$ or healthy obese group $(\mathrm{p}=0.006)$ (Figure 3A). In 
Table 2 Metabolism-Related Comorbidities in Normal or Hypercholesterolemia

\begin{tabular}{|c|c|c|c|c|}
\hline & \multicolumn{2}{|c|}{ Control Group } & \multicolumn{2}{|c|}{ XP Group } \\
\hline & Normal (28) & Hyper (13) & Normal (I I) & Hyper (25) \\
\hline Age (mean; IQR, years) & $53.1 ; 15.5$ & $56.5 ; 11$ & $56.2 ; 11$ & $58.7 ; 12$ \\
\hline Body mass index (mean; IQR) & 22.99; 3.09 & $22.4 ; 6.4$ & $25.42 ; 5.8$ & $25.55 ; 2.66 *$ \\
\hline Coronary artery disease (n) & 0 & 0 & 3 & 7 \\
\hline Type 2 diabetes mellitus ( $n$ ) & 3 & 0 & 1 & 7 \\
\hline Stroke $(n)$ & 0 & 0 & 0 & 0 \\
\hline Total cholesterol (mean; IQR, mg/dL) & $166.25 ; 30.5$ & $240.62 ; 48.5^{*}$ & 170.36; 35 & $239.32 ; 45.5^{*}$ \\
\hline Low density lipoprotein (mean; IQR, mg/dL) & $100.14 ; 22$ & $150.85 ; 30.5^{*}$ & $111.55 ; 17^{*}$ & $156.72 ; 44^{*}$ \\
\hline High density lipoprotein (mean; IQR, mg/dL) & $50.86 ; 15$ & $58.46 ; 25$ & $42.67 ; 15$ & $50.42 ; 25$ \\
\hline Triglyceride (mean; IQR, mg/dL) & II $2.89 ; 58$ & $171.54 ; 125$ & $109.73 ; 76$ & $176.13 ; 103.5^{*}$ \\
\hline Hepatic steatosis index (mean; IQR) & $32.33 ; 4.94$ & $31.21 ; 9$ & $35.6 ; 5.7$ & $37.7 ; 7.5^{*}$ \\
\hline ZJU index (mean; IQR) & $32.97 ; 5.5 I$ & $33.98 ; 7.7$ & $37.65 ; 6.8^{*}$ & $39.37 ; 7.64 *$ \\
\hline
\end{tabular}

Notes: Patients with and without xanthelasma palpebrarum were divided into subgroups with normal or hypercholesterolemia. Demographic data, lipid profiles, and evaluation scores for non-alcoholic fatty liver disease, such as the hepatosteatosis index (HSI) and ZJU index were compared. * $\mathrm{p}<0.05$, compared to the control normal cholesterolemia group.

Abbreviation: IQR, interquartile range.

GSE61260, people with NAFLD presented with higher $Z$ scores than those in the normal control group ( $\mathrm{p}=0.004)$; people with NASH also had higher $Z$ scores than those in the normal control group $(\mathrm{p}=0.017)$ (Figure 3B).

\section{Identifying the Potential Significance of Xanthelasma-Associated Genes Using GSVA Scores}

To determine the genetic background of XP, we used the GSVA method to establish gene significance in patients with XP. A total of eight genes, ABCG518, ABCG519, LDLRAP120, PCSK921, APOA121, ALOX522, ABCG823, ABCG824, LDLR25-27, and APOE28 were identified (Figure 4B). Cardiovascular diseases, XP, and lipid disorders all shared similar genetic backgrounds (Figure 4A).

\section{Discussion}

Xanthelasma palpebrarum is a benign condition that rarely has serious consequences, but it affects the cosmetic appearance of the patient. People with XP usually seek medical advice and visit dermatology, plastic surgery, and ophthalmology clinics to find solutions. Given that about $50 \%$ of people with XP develop lipid disorders, ${ }^{31}$ and most of them seek only cosmetic management, it is worthwhile to discuss the risk of cardiovascular events and fatty liver in individuals with XP.

In our study, the majority of patients were females presenting with cosmetic problems, which is consistent with other studies. ${ }^{1,32}$ However, the effect of sex on morbidity has not been determined, as other studies have also reported a high morbidity rate in men. ${ }^{33}$ The fact that women are more conscious of their appearance than men may explain the higher incidence in women in some studies. In our study, patients with XP had heavier weight and higher body mass index, which are also risk factors for cardiovascular disease. ${ }^{34}$ A high body mass index could help explain why patients with XP are more likely to have a higher incidence of CAD. Of clinical importance, we also explicitly established XP as a predictor of NAFLD using HSI and ZJU indices as quantitative strategies (Table 1). In our cohort study, although patients with XP did not show elevated levels of hepatitis markers, such as AST and ALT, a higher risk of fatty liver disease could be predicted. Because these patients were relatively young (58.14 \pm 2.0 years), even the progression to steatohepatitis may still be in the early stages of the optimal period of treatment.

In our study, the subgroup of patients with XP who also had hypercholesterolemia showed higher scores on 

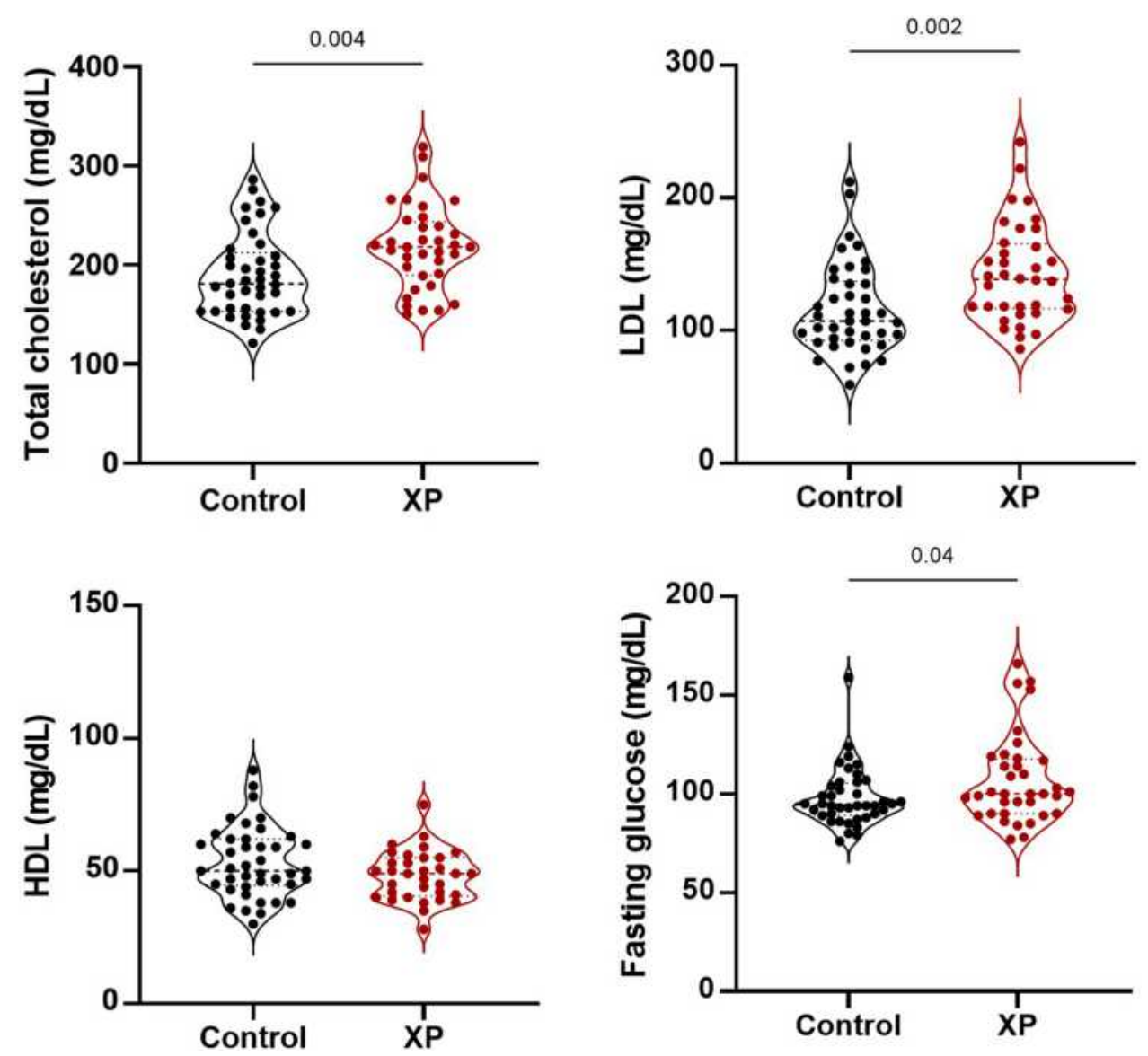

Figure 2 Comparison between patients with or without xanthelasma palpebrarum under fatty liver conditions. The lipid profiles between patients with and without XP were compared in the setting of fatty liver. The diagnosis of fatty liver was based on sonographic findings.

Abbreviations: LDL, low-density lipoprotein; HDL, high-density lipoprotein; XP, xanthelasma palpebrarum.

A

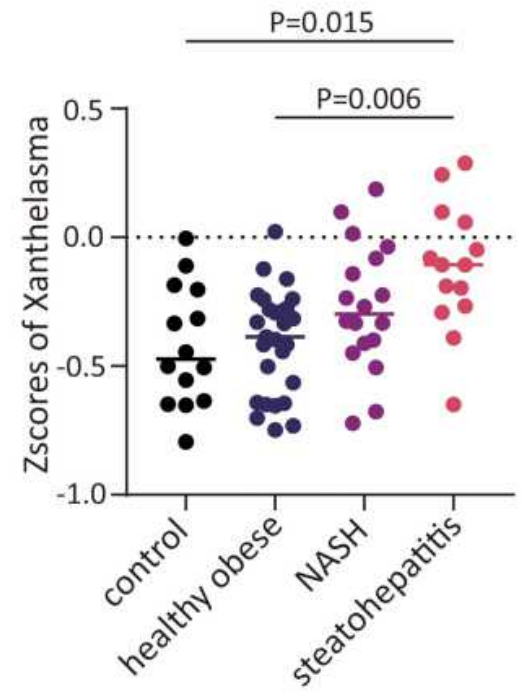

B
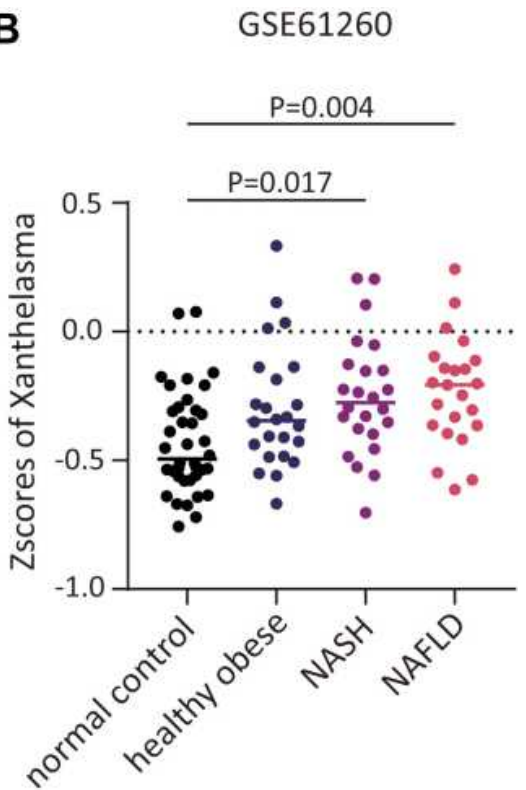

Figure 3 Relationship between xanthelasma palpebrarum and fatty liver. Functional enrichment analysis revealed an association between xanthelasma and fatty liver disease, non-alcoholic fatty liver disease (NAFLD), and non-alcoholic steatohepatitis (NASH). Databank from GSE48452 (A) and GSE6I260 (B). 
A

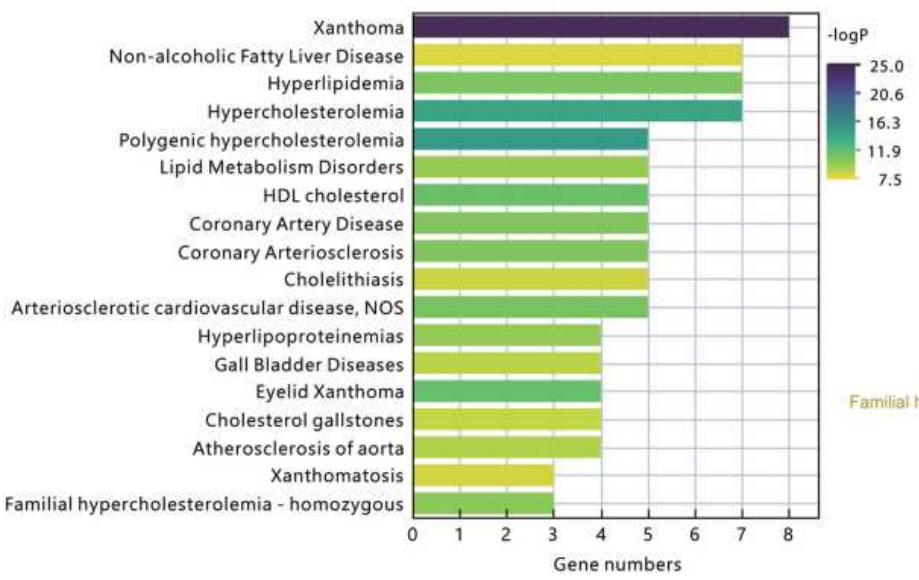

B

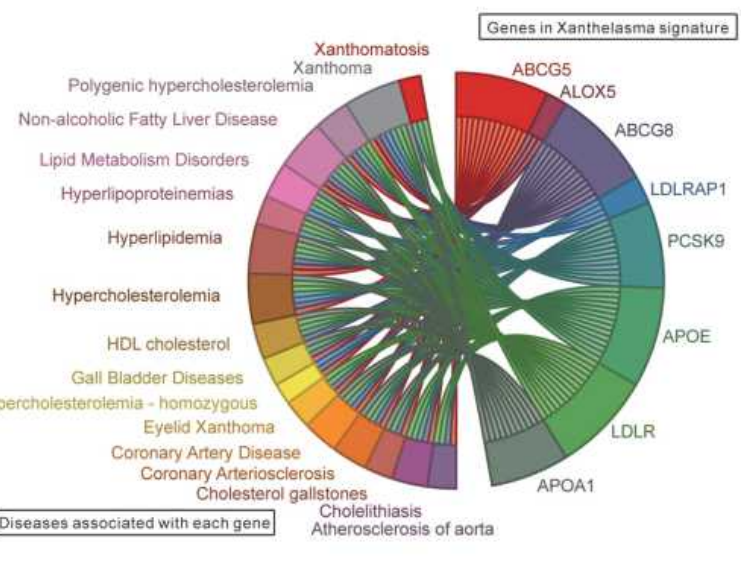

Figure 4 Gene significance of xanthelasma by calculation of GSVA scores. To establish the genetic background of xanthelasma, GSVA scores were calculated, and eight genes, including ABCG5, LDLRAPI, PCSK9, APOAI, ALOX5, ABCG8, LDLR, and APOE, which are related to xanthelasma, were identified (B). Association of xanthelasmarelated genes with cardiovascular disease or other lipid-related diseases were also investigated (A).

the HSI and ZJU indices, as expected. In particular, the assessed scores on the ZJU index $(37.65 \pm 1.52 \cdot$ vs $32.97 \pm$ $\cdot 1.19, \mathrm{p}=0.045)$ were higher in patients with XP than in those without XP, even though they had normal serum total cholesterolemia levels (Table 2). Since XP patients with normal serum total cholesterol levels also showed higher serum LDL concentrations, disturbances in lipoprotein metabolism may explain why these individuals still have a higher predicted risk of fatty liver. ${ }^{35,36}$

By comparing characteristic differences in patients with fatty liver, we found higher serum total cholesterol, LDL, and fasting glucose levels in the XP group. Although markers of NAFLD (HSI, ZJU, and FIB-4 scores) did not differ significantly, patients with XP still showed a trend towards higher scores in these indicators. These findings support XP as a predictor for the development of more metabolic comorbidities in fatty liver disease (Figure 2).

To examine which component of lipoprotein is the most sensitive lipid marker, we measured lipid profiles and found that total cholesterol and LDL were the most susceptible markers in patients with XP. In line with this, previous studies have also shown that total cholesterol and LDL are the most sensitive markers. ${ }^{28,37-39}$

Among the enrolled cases, we found that up to $38.3 \%$ of patients with XP sought cosmetic advice only (23/60, Figure 1). After the treatment of XP, they did not undergo any lipidrelated disease assessment and serum lipid screening, suggesting that the risk of cardiovascular events and NAFLD is underestimated in these patients. This also highlights that the health issues associated with the cosmetic problems of XP may need more attention.
This retrospective study has some limitations. For example, the patients with XP may not be new cases and may have already been undergoing cholesterollowering therapy. The risk and incidence rates in this study may be lower than they actually are because we only investigated patients who lived in the capital region, where people are usually better off financially. In addition, people from the medical examination department may not be the best control group. Furthermore, a larger population size is needed conclude because of the small number of XP cases.

To find more evidence, we used the clinical liver diseaseassociated gene expression datasets, GSE48452 and GSE61260, to analyze the relationship between NAFLD/ NASH and xanthelasma-associated gene expression. Patients with NAFLD and NASH had higher xanthelasma GSVA scores, supporting our clinical findings in patients with XP.

Using the GSVA strategy for specific gene-disease enrichment analysis, we further identified the association of the overall expression of potential genes associated with cardiovascular disease and lipid disorders, including ABCG5, LDLRAP1, PCSK9, APOA1, ALOX5, ABCG8, LDLR, and APOE. This analysis based on human samples is believed to be convincing. A better understanding of the correlation between xanthelasma and fatty liver can be obtained based on the clinical presentation and genetic background of patients.

Taken together, our results reveal a potential risk of NAFLD in patients with XP and suggest an underestimated incidence of lipid-related comorbidities associated with cosmetic problems. Screening for fatty liver should be considered in patients with XP. 


\section{Acknowledgment}

We thank all the patients who participated in this study.

\section{Author Contributions}

All authors made a significant contribution to the work reported, in terms of conception, study design, execution, acquisition of data, analysis and interpretation, or areas all of these; took part in drafting, revising, or critically reviewing the article; gave final approval of the version to be published; have agreed on the journal to which the article has been submitted; and agree to be accountable for all aspects of the work.

\section{Funding}

This study was supported in part by grants from the Foundation for Medical Research of Tri-Service General Hospital, Taiwan (R.O.C.) (TSGH-E-110264 to HW Chen) and the Ministry of Science and Technology, Taiwan (R.O. C.) (MOST 107-2314-B-016-001- to J.-C. LIN; MOST109-2320-B-016-004- to Y.-L. CHIU).

\section{Disclosure}

The authors report no conflicts of interest in this work.

\section{References}

1. Vacca JB, Knight WA Jr, Broun GO Sr. Clinical observations regarding xanthelasma. Ann Intern Med. 1959;51:1019-1031.

2. Jain A, Goyal P, Nigam PK, Gurbaksh H, Sharma RC. Xanthelasma Palpebrarum-clinical and biochemical profile in a tertiary care hospital of Delhi. Indian J Clin Biochem. 2007;22(2):151-153. doi:10.1007/ BF02913335

3. Gordon T, Castelli WP, Hjortland MC, Kannel WB, Dawber TR. High density lipoprotein as a protective factor against coronary heart disease. The Framingham Study. Am J Med. 1977;62(5):707-714. doi:10.1016/0002-9343(77)90874-9

4. Harada-Shiba M, Ako J, Arai H, et al. Prevalence of familial hypercholesterolemia in patients with acute coronary syndrome in Japan: results of the EXPLORE-J study. Atherosclerosis. 2018;277:362-368. doi:10.1016/j.atherosclerosis.2018.06.856

5. Pandhi D, Gupta P, Singal A, Tondon A, Sharma S, Madhu SV. Xanthelasma palpebrarum: a marker of premature atherosclerosis (risk of atherosclerosis in xanthelasma). Postgrad Med J. 2012;88 (1038):198-204. doi:10.1136/postgradmedj-2011-130443

6. Chang HC, Sung CW, Lin MH. Serum lipids and risk of atherosclerosis in xanthelasma palpebrarum: a systematic review and meta-analysis. $J$ Am Acad Dermatol. 2020;82(3):596-605. doi:10.1016/j.jaad.2019.08.082

7. Bergman R. Xanthelasma palpebrarum and risk of atherosclerosis. Int $J$ Dermatol. 1998;37(5):343-345. doi:10.1046/j.13654362.1998.00362.x

8. Watanabe A, Yoshimura A, Wakasugi T, et al. Serum lipids, lipoprotein lipids and coronary heart disease in patients with xanthelasma palpebrarum. Atherosclerosis. 1981;38(3-4):283-290. doi:10.1016/ 0021-9150(81)90044-7

9. Laftah Z, Al-Niaimi F. Xanthelasma: an update on treatment modalities. J Cutan Aesthet Surg. 2018;11(1):1-6. doi:10.4103/JCAS. JCAS_56_17
10. Chalasani N, Younossi Z, Lavine JE, et al. The diagnosis and management of nonalcoholic fatty liver disease: practice guidance from the American association for the study of liver diseases. Hepatology. 2018;67(1):328-357.

11. Subichin M, Clanton J, Makuszewski M, Bohon A, Zografakis JG, Dan A. Liver disease in the morbidly obese: a review of 1000 consecutive patients undergoing weight loss surgery. Surg Obes Relat Dis. 2015;11(1):137-141. doi:10.1016/j.soard.2014.06.015

12. Leite NC, Salles GF, Araujo AL, Villela-Nogueira CA, Cardoso CR. Prevalence and associated factors of non-alcoholic fatty liver disease in patients with type-2 diabetes mellitus. Liver Int. 2009;29 (1):113-119. doi:10.1111/j.1478-3231.2008.01718.x

13. Lee JH, Kim D, Kim HJ, et al. Hepatic steatosis index: a simple screening tool reflecting nonalcoholic fatty liver disease. Dig Liver Dis. 2010;42(7):503-508. doi:10.1016/j.dld.2009.08.002

14. Wang J, Xu C, Xun Y, et al. ZJU index: a novel model for predicting nonalcoholic fatty liver disease in a Chinese population. Sci Rep. 2015;16(5):16494. doi:10.1038/srep16494

15. Ahrens M, Ammerpohl O, von Schönfels W, et al. DNA methylation analysis in nonalcoholic fatty liver disease suggests distinct disease-specific and remodeling signatures after bariatric surgery. Cell Metab. 2013;18(2):296-302. doi:10.1016/j.cmet.2013.07.004

16. Horvath S, Erhart W, Brosch M, et al. Obesity accelerates epigenetic aging of human liver. Proc Natl Acad Sci U S A. 2014;111 (43):15538-15543. doi:10.1073/pnas.1412759111

17. Hanzelmann S, Castelo R, Guinney J. GSVA: gene set variation analysis for microarray and RNA-seq data. BMC Bioinform. 2013;14(1):7. doi:10.1186/1471-2105-14-7

18. Pinero J, Ramirez-Anguita JM, Sauch-Pitarch J, et al. The DisGeNET knowledge platform for disease genomics: 2019 update. Nucleic Acids Res. 2020;48(D1):D845-D855. doi:10.1093/nar/gkz1021

19. Bastida JM, Benito R, Janusz K, et al. Two novel variants of the ABCG5 gene cause xanthelasmas and macrothrombocytopenia: a brief review of hematologic abnormalities of sitosterolemia. J Thromb Haemost. 2017;15(9):1859-1866. doi:10.1111/jth.13777

20. Huang D, Zhou Q, Chao YQ, Zou CC. Clinical features and genetic analysis of childhood sitosterolemia: two case reports and literature review. Medicine (Baltimore). 2019;98(15):e15013. doi:10.1097/ MD.0000000000015013

21. Tada H, Kawashiri MA, Ohtani R, et al. A novel type of familial hypercholesterolemia: double heterozygous mutations in LDL receptor and LDL receptor adaptor protein 1 gene. Atherosclerosis. 2011;219(2):663-666. doi:10.1016/j.atherosclerosis.2011.08.004

22. Saavedra YG, Dufour R, Davignon J, Baass A. PCSK9 R46L, lower LDL, and cardiovascular disease risk in familial hypercholesterolemia: a cross-sectional cohort study. Arterioscler Thromb Vasc Biol. 2014;34(12):2700-2705. doi:10.1161/ATVBAHA.114.304406

23. Oosterveer DM, Versmissen J, Yazdanpanah M, et al. 5-Lipoxygenase activating protein (ALOX5AP) gene variants associate with the presence of xanthomas in familial hypercholesterolemia. Atherosclerosis. 2009;206(1):223-227. doi:10.1016/j.atherosclerosis.2009.02.019

24. Bardawil T, Rebeiz A, Chaabouni M, et al. Mutations in the ABCG8 gene are associated with sitosterolaemia in the homozygous form and xanthelasmas in the heterozygous form. Eur J Dermatol. 2017;27 (5):519-523. doi:10.1684/ejd.2017.3087

25. Hu M, Yuen YP, Kwok JS, Griffith JF, Tomlinson B. Potential effects of NPC1L1 polymorphisms in protecting against clinical disease in a chinese family with sitosterolaemia. $J$ Atheroscler Thromb. 2014;21 (9):989-995. doi:10.5551/jat.24679

26. Mabuchi H. Half a century tales of Familial Hypercholesterolemia (FH) in Japan. $J$ Atheroscler Thromb. 2017;24(3):189-207. doi:10.5551/jat.RV16008

27. Gaspar IM, Gaspar A. Variable expression and penetrance in Portuguese families with familial hypercholesterolemia with mild phenotype. Atheroscler Suppl. 2019;36:28-30. doi:10.1016/j. atherosclerosissup.2019.01.006 
28. Donato LJ, Saenger AK, Train LJ, et al. Genetic and biochemical analyses in dyslipidemic patients undergoing LDL apheresis. J Clin Apher. 2014;29(5):256-265. doi:10.1002/jca.21317

29. Tursen U, Eskandari G, Kaya TI, Tamer L, Ikizoglu G, Atik U. Apolipoprotein E polymorphism and lipoprotein compositions in normolipidaemic xanthelasma patients. J Eur Acad Dermatol Venereol. 2006;20(3):260-263. doi:10.1111/j.1468-3083.2006.01418.x

30. Chen J, Bardes EE, Aronow BJ, Jegga AG. ToppGene Suite for gene list enrichment analysis and candidate gene prioritization. Nucleic Acids Res. 2009;37:W305-11. doi:10.1093/nar/gkp427

31. $\mathrm{Su} \mathrm{HC}$, Wu SC, Yen LC, et al. Gene expression profiling identifies the role of Zac1 in cervical cancer metastasis. Sci Rep. 2020;10 (1):11837. doi:10.1038/s41598-020-68835-0

32. Al Aboud AM, Al Aboud DM. Xanthelasma Palpebrarum. Updated 2020 Aug 10. In: StatPearls [Internet]. Treasure Island (FL): StatPearls Publishing; 2021.Available from https://www.ncbi.nlm. nih.gov/books/NBK531501/. Accessed April 30, 2021.

33. Nair PA, Patel CR, Ganjiwale JD, Diwan NG, Jivani NB. Xanthelasma palpebrarum with arcus cornea: a clinical and biochemical Study. Indian J Dermatol. 2016;61(3):295-300. doi:10.4103/ 0019-5154.182426
34. Dey A, Aggarwal R, Dwivedi S. Cardiovascular profile of xanthelasma palpebrarum. Biomed Res Int. 2013;2013:932863. doi:10.1155/ 2013/932863

35. Khan SS, Ning H, Wilkins JT, et al. Association of body mass index with lifetime risk of cardiovascular disease and compression of morbidity. JAMA Cardiol. 2018;3(4):280-287. doi:10.1001/ jamacardio.2018.0022

36. Sugino I, Kuboki K, Matsumoto T, Murakami E, Nishimura C, Yoshino G. Influence of fatty liver on plasma small, dense LDLcholesterol in subjects with and without metabolic syndrome. J Atheroscler Thromb. 2011;18(1):1-7. doi:10.5551/jat.5447

37. Toledo FG, Sniderman AD, Kelley DE. Influence of hepatic steatosis (fatty liver) on severity and composition of dyslipidemia in type 2 diabetes. Diabetes Care. 2006;29(8):1845-1850. doi:10.2337/dc060455

38. Ozdol S, Sahin S, Tokgozoglu L. Xanthelasma palpebrarum and its relation to atherosclerotic risk factors and lipoprotein. Int J Dermatol. 2008;47(8):785-789. doi:10.1111/j.1365-4632.2008.03690.x

39. Noel B. Premature atherosclerosis in patients with xanthelasma. J Eur Acad Dermatol Venereol. 2007;21(9):1244-1248. doi:10.1111/ j.1468-3083.2007.02252.x
Journal of Inflammation Research

\section{Publish your work in this journal}

The Journal of Inflammation Research is an international, peerreviewed open-access journal that welcomes laboratory and clinical findings on the molecular basis, cell biology and pharmacology of inflammation including original research, reviews, symposium reports, hypothesis formation and commentaries on: acute/chronic inflammation; mediators of inflammation; cellular processes; molecular

\section{Dovepress}

mechanisms; pharmacology and novel anti-inflammatory drugs; clinical conditions involving inflammation. The manuscript management system is completely online and includes a very quick and fair peerreview system. Visit http://www.dovepress.com/testimonials.php to read real quotes from published authors. 